\title{
The sum is more complex than its parts
}

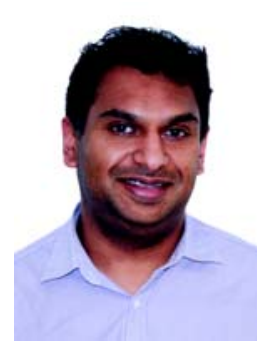

Astika Kappagoda Deputy Editor

mja@mja.com.au

doi: 10.5694/mjal3.c0415 single nutrients leads to distorted food choices, and taking nutrients as supplements does not allow for the complex interaction between nutrients in whole food, which may lead to adverse consequences.

These issues are reflected in Brimblecombe and colleagues' survey of diets in remote Northern Territory Indigenous communities (page 380). They note high intakes of sugary drinks and processed foods fortified with nutrients, alongside low fruit and vegetable intakes. How does this relate to the high burden of chronic disease in these communities? We need to look further than body mass index and total kilojoule and nutrient intake to fully explain the connections between diet and health.

An overly reductionist approach to disease assumes that tinkering with single environmental factors or isolated physiological and biochemical processes will substantially affect wellness or illness. A large section of the food industry and the entire pharmaceutical industry are built on this assumption, which is often justified. But according to complex-systems science, altering one factor can change a system in unpredictable ways. In health terms, this can manifest as either increased risk of other diseases or adverse events. Studying single factors makes for clearly defined research but neglects the complexity of the human body in its environment.

Such complexity has been recognised in other areas. Our understanding of communicable illness has moved well beyond "microbe A causes disease X"; earlier, the "father of pathology" Rudolf Virchow was, unsurprisingly, inexorably led to investigate connections between causative agents, illness and the social environment (Am J Pub Health 2006; 96: 2104-2105). Larney and colleagues' cross-sectional study of susceptibility to vaccine-preventable diseases in prison inmates (page 376) exemplifies the complexity of communicable diseases: the socioeconomic and cultural backgrounds and the lifestyles of inmates, modes of disease transmission in correctional facilities, and opportunities for intervention all need to be considered when devising health strategies for this vulnerable population who are "racked, stacked and packed" in the wider community's mindset. Kirby (page 355) highlights the complex social and administrative issues in Papua New Guinea (PNG) that have led to doctors in Australia having to confront extensively drug-resistant tuberculosis, which is difficult to manage, requiring prolonged treatment and expensive resources. A PNG national died in Queensland this year after lengthy treatment for this disease.

Even assessing the therapeutic benefit and safety profile of pharmacological agents - the archetypal single-agent intervention - is often complex and controversial. Attia and Pearce (page 356) and Eikelboom and Hankey (page 358) dissect the results of the RE-LY (Randomized Evaluation of Long-Term Anticoagulation Therapy) clinical trial of the anticoagulant dabigatran and come to somewhat different conclusions. The interaction of selection bias and confounding effects from co-interventions and comorbidities are prominent, as they often are when considering drugs used for chronic diseases.

In future, to promote wellness and devise lasting solutions to disease, we will need to better understand the complexity underpinning both. The challenge is for medicine and medical research to put aside single-factor thinking and regard humans as the complex systems we are, recognising that we are not merely a collection of parts that can be targets for silver bullets.

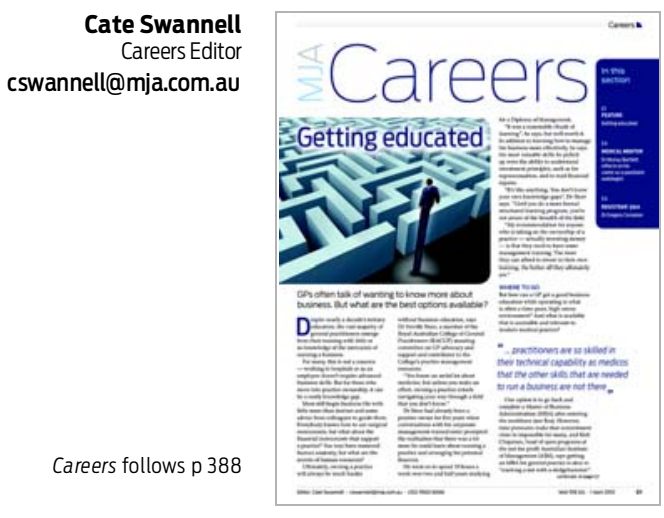

Te Swannell
Careers Editor wannell@mja.com.au

\section{Getting down to business}

Despite nearly a decade of tertiary education, most general practitioners emerge from their training with little or no knowledge of the intricacies of running a business. For those moving into practice ownership, this knowledge gap could prove costly. Annabel McGilvray speaks with Dr Neville Steer from the Royal Australian College of General Practitioners standing committee on GP advocacy and support, and Rick Chapman from the not-for-profit Australian Institute of Management about the existing options and educational opportunities for practice owners (pages C1 and C2). Dr Murray Bartlett was a paediatric physician for 5 years, before deciding to make the switch to radiology. He talks with Karen Burge about the joy he finds in his involvement with a diverse range of cases, and as a mentor of younger doctors (page C4). The registrar under our spotlight in this edition is Dr Gregory Compton, who has worked under Dr Bartlett at Royal Children's Hospital in Melbourne (page C4). 
Editor's choice

348 MJA $198(7) \cdot 15$ April 2013 